\title{
Über die Geschwindigkeit der Auflösung von Zinkblende und Bleiglanz in verdünnter Schwefelsäure.
}

\author{
Von \\ Felix Rosenkränzer. \\ Mit 4 Figuren in Text.
}

Im Zusammenhang mit Versuchen über die Theorie der Flotationsprozesse wurde auch die Frage nach der Angreifbarkeit sulfidischer Erze durch verdünnte Mineralsäuren, insbesondere durch verdünnte Schwefelsäure, aufgerollt und an Zinkblende- und Bleiglanzproben einer experimentellen Untersuchung unterworfen. Über die Ergebnisse dieser Untersuchung möge, da sie für die Kenntnis der Reaktionsgeschwindigkeit in heterogenen Systemen ron einigem Interesse sind, im folgenden kurz berichtet werden.

\section{Experimentelles.}

Als Versuchsmaterial dienten vier Zinkblenden von verschiedener Herkunft sowie Clausthaler Bleiglanz. ${ }^{1}$ Die nötigen Angaben über Herkunft und Zusammensetzung der Erze sind in Tabelle 1 zusammengestellt.

Tabelle 1.

\begin{tabular}{|c|c|c|c|c|c|c|c|c|c|c|c|c|}
\hline & $\begin{array}{l}\text { Bezeichnung } \\
\text { u. Herkunft } \\
\text { des Erzes }\end{array}$ & $\begin{array}{c}\text { Mineralog. } \\
\text { Ausseben }\end{array}$ & $\mathrm{SiO}_{\mathbf{2}}$ & $\mathrm{Pb}$ & $\begin{array}{l}\text { Chemi } \\
\text { Cd }\end{array}$ & $\begin{array}{c}\text { sche } \mathrm{Z} \\
\mathrm{Cu}\end{array}$ & Fe & $\begin{array}{l}\text { mensetz } \\
\left|Z_{n}\right|\end{array}$ & $\begin{array}{l}\text { zung: } \\
\mid \mathbf{M n} \text { : }\end{array}$ & $\mathrm{CaO}$ & $\mathrm{S}$ & $\begin{array}{c}\text { Summa } \\
\%\end{array}$ \\
\hline 1 & $\begin{array}{l}\text { Clausthaler } \\
\text { Zinkblende }\end{array}$ & $\begin{array}{c}\text { derb, } \\
\text { dunkelbraun }\end{array}$ & 2.87 & 11.41 & 0.09 & 0.50 & 3.59 & 52.52 & 0.18 & - & 28.77 & 99.93 \\
\hline 2 & $\left\{\begin{array}{c}\text { Clausthaler } \\
\text { Bleiglanz }\end{array}\right.$ & $\begin{array}{c}\text { derb, röt- } \\
\text { lichbleigrau }\end{array}$ & 0.32 & $85.71^{2}$ & - & - & 0.20 & 0.51 & - & - & $13.26^{2}$ & 100.00 \\
\hline 3 & $\left\{\begin{array}{c}\text { Blende v. Pi- } \\
\text { cos de Europa, } \\
\text { Spanien }\end{array}\right.$ & $\left|\begin{array}{c}\text { hellgelb u. halb- } \\
\text { durchsichtig, sog. } \\
\text { Honigbiendo }\end{array}\right|$ & 0.20 & - & - & - & 0.57 & $66.54^{2}$ & - & - & $32.69^{2}$ & 100.00 \\
\hline 4 & $\left\{\begin{array}{c}\text { Blende der } \\
\text { Grube Berzeliug } \\
\text { bei Bensberg }\end{array}\right.$ & $\begin{array}{l}\text { derb, dunkel- } \\
\text { braun ins blei- } \\
\text { graue }\end{array}$ & 11.00 & 14.89 & Spur & - & 3.45 & 43.43 & 0.36 & 0.88 & 25.03 & 99.04 \\
\hline 5 & $\left\{\begin{array}{c}\text { Christophit v. } \\
\text { Breitenbrunn } \\
\text { i/Sa. }\end{array}\right.$ & $\begin{array}{l}\text { sqmtschwarz, } \\
\text { spröd a. ãub. } \\
\text { feinverwachs. }\end{array}$ & 18.97 & Spur. & 0.20 & Spur. & 16.00 & 36.15 & $|0.98|$ & $1-$ & 27.65 & 89.95 \\
\hline
\end{tabular}

${ }_{1}$ Den Direktionen der Gruben Berzelius bei Bensberg und St. Christoph bei Breitenbrunn $\mathrm{i} / \mathrm{Sa}$. bin ich für die freundliche Zusendung des Versucbsmaterials zu Dank verpflichtet.

2 Diese Zahlen sind nur aus der Differenz bestimmt. 
Durch mechanische Zerkleinerung und Siebung wurden die Erze in Fraktionen von verschiedener KorngröBe zerlegt und der Durchmesser der Körner gleich dem arithmetischen Mittel aus den lichten Maschenweiten der beiden Siebe gesetzt, zwischen die die Fraktion eingeschlossen war. Dann wurden abgewogene Mengen der Fraktionen mit abgemessenen Mengen verdünnter $(1.25 \%$ iger, $0.125 \%$ iger oder $0.0125 \%$ iger) Schwefelsäure bei konstanter Temperatur während einer bestimmten Zeit geschüttelt, und schlieBlich die Menge des in die Flüssigkeit übergegangenen Schwefelwasserstoffs ermittelt. Die Einwirkung der Schwefelsäure auf die Erze geschah in Flaschen aus weiBem Glase, die mit gut passenden Kautschukstopfen verschlossen waren. Da die entwickelte Schwefelwasserstoffmenge in allen Fällen nur sehr gering und die Flaschen fast vollständig mit der verdünnten Säure angefüllt waren - auf $400 \mathrm{ccm}$ Flüssigkeit kann ein Luftvolumen von nur etwa $4 \mathrm{ccm}-$, so kounten die Spuren von Schwefelwasserstoff, die in den Gasraum übergingen und bei den Analysen nicht mitgemessen wurden, unbedenklich vernachlässigt werden. Bei den bei Zimmertemperatur ausgeführten Versuchen wurden die Proben in einem Schüttelapparat mäBig rasch (40-50 Umdrehungen in der Minute) geschüttelt, bei den bei anderer Temperatur durchgeführten Versuchen, bei denen die Flaschen in schmelzendem Eise oder in GefäBen mit warmem Wasser von konstant gehaltener Temperatur standen, wurde nur in angemessenen Zeitabständen mit der Hand geschüttelt. Kontrollversuche bei Zimmertemperatur ergaben, daß beim Schütteln mit der Hand und beim Schütteln im Schüttelapparat gleiche Resultate erhalten werden.

Die Menge des bei der Reaktion entstandenen Schwefelwasserstoffs wurde nach einem bereits früher beschriebenen Verfahren ${ }^{1}$ durch Umwandlung des in aliquoten Teilen der Reaktionslösung enthaltenen Schwefelwasserstoffs in Methylenblau, und dessen kolorimetrische Auswertung im WoLfFschen Kolorimeter ermittelt. Der dieser bis zu einer Grenzrerdünnung von etwa 1:100 Millionen (0.01 mg $=10 \mu \mathrm{g} \mathrm{H}_{2} \mathrm{~S} /$ lit) brauchbaren Bestimmungsmethode anhaftende relative Fehler beträgt im allgemeinen nicht mehr als $2 \%$ und steigt erst bei einer Verdünnung ron etwa $1: 20$ Millionen

1 Werser Mecklendora u. Felix Rosenkränzer, Über eine kolorimetrische Methode zur Bestimmung kleiner Sehwefelwasserstoffmengen; Z. anorg. Chem. 86 (1914), $143 \mathrm{ff}$. 
(50 $\mu \mathrm{g} \mathrm{H}_{2} \mathrm{~S} /$ lit) und darunter auf etwa $\pm 10 \%$. Wegen aller Einzelheiten des Verfahrens sei auf die obengenannte Arbeit verwiesen.

Da eine durch das Schütteln hervorgebrachte, wenn auch geringe Trübung der Reaktionslösungen sich beim Kolorimetrieren recht unangenehm bemerkbar machte - selbst über Nacht schwand sie nicht völlig —, so wurden die Erze, bevor sie zu den Versuchen Verwendung fanden, gründlich mit Wasser gewaschen; die störende Trübung trat dann nicht mehr auf.

Zunächst wurden Versuche mit Clausthaler Blende angestellt, wobei sich folgende GesetzmäBigkeiten zeigten:

1. Die entwickelte Schwefelwasserstoffmenge ist direkt proportional der Zeitdauer der Reaktion, oder mit andern Worten, die Reaktionsgeschwindigkeit bleibt bis zum Ende des Versuchs konstant (vgl. Tabelle 2 u. 3, sowie 4, 5, 6 u. 7 . Aus diesen Tabellen sind auch alle notwendigen Angaben über die angewandten $\mathrm{H}_{2} \mathrm{SO}_{4}$-Konzentrationen, über Korndurchmesser, Zeit und Temperatur zu ersehen.)

2. Der Angriff der Sulfide ist der Schwefelsäurekonzentration direkt proportional. Von $1.25 \%$ iger Schwefelsäure wird die Blende zehnmal so stark angegriffen wie von $0.125 \%$ iger, und von $0.125 \%$ iger wieder zehnmal so stark wie von $0.0125 \%$ iger Säure (vgl. Tabelle 4, 5, 6 u. 7).

3. Bildet man das Verhältnis zweier unter gleichen Bedingungen aus Korngröße 1 bzw. 2 und KorngröBe 3 entwickelten Schwefelwasserstoffmengen, so zeigt sich, daB dieses Verhältnis konstant und zwar gleich dem umgekehrten Verhältnis der Quadrate der Korndurchmesser ist (vgl. Tabelle 8 u. 9). Mit anderen Worten: Die entwickelten Schwefelwasserstoffmengen sind der Ober-

Tabelle 2.

Aus $10 \mathrm{~g} \mathrm{ZnS} \mathrm{der} \mathrm{KorngröBe} 2$ (Durchmesser $0.90 \mathrm{~mm}$ ) wurden von $1.25 \%$ iger $\mathrm{H}_{2} \mathrm{SO}_{4}$ entwickelt bei $\vartheta=20^{\circ} \mathrm{C}$ :

\begin{tabular}{c|c|c}
\hline Nach Stunden & $\mu \mathrm{g} \mathrm{H}_{2} \mathrm{~S}$ & $\begin{array}{c}\text { Reaktionsgeschwindigkeit } \\
\mu \mathrm{g}_{2} \mathrm{~S} / \mathrm{Std} .\end{array}$ \\
\hline \hline $1 / 2$ & 21 & 42.0 \\
1 & 42 & 42.0 \\
$1^{1 / / 2}$ & 64 & 42.7 \\
2 & 85 & 42.5 \\
$2^{1 / 2}$ & 106 & 42.4 \\
3 & 129 & 43.0 \\
$3^{1 / 2}$ & 148 & 42.2 \\
4 & 169 & 42.3 \\
$4^{1 / 2}$ & 191 & 42.4 \\
\hline
\end{tabular}


fläche der Zinkblendekörner direkt proportional. Bestätigt wird dies Ergebnis besonders durch die später bei verschiedener Temperatur und auch mit wechselnden absoluten Mengen ${ }^{1}$ ausgeführten Versuche, deren Ergebnisse weiter unten in Tabelle 13 und 14 aufgeführt sind; auch hier zeigt sich Proportionalität zwischen der Stärke des Angriffs und der Größe der Oberfläche.

Tabelle 3 .

Aus $10 \mathrm{~g} \mathrm{ZnS} \mathrm{der} \mathrm{KorngröBe} 1$ (Durchmesser $=1.13 \mathrm{~mm}$ ) wurden entwickelt bei $80^{\circ}$ :

\begin{tabular}{|c|c|c|c|c|c|}
\hline $\begin{array}{l}\text { Nach } \\
\text { Stdn. }\end{array}$ & \multicolumn{2}{|c|}{$\begin{array}{c}\text { Von } \\
1.25 \% \text { iger } \mathrm{H}_{2} \mathrm{SO}_{4}\end{array}$} & \multicolumn{2}{|c|}{$\begin{array}{c}\text { Von } \\
0.125 \% \text { iger } \mathrm{H}_{2} \mathrm{SO}_{4}\end{array}$} & \multirow[t]{2}{*}{$\begin{array}{c}\text { Von } \\
0.0125 \% \text { iger } \mathrm{H}_{2} \mathrm{SO}_{4}\end{array}$} \\
\hline \multirow{11}{*}{$\begin{array}{l}1^{1 / 2} \\
1^{1 / 2} \\
2 \\
2^{1 / 2} \\
3 \\
3^{1 / 2} \\
4 \\
4^{1 / 2}\end{array}$} & $\mathrm{H}_{2} \mathrm{~S}$ & $\mathrm{H}_{2} \mathrm{~S}$ & $\mathrm{H}_{2} \mathrm{~S}$ & $\mathrm{H}_{2} \mathrm{~S}$ & \\
\hline & $180 \mu$ & $=360 \mu \mathrm{g} / \mathrm{Std}$. & 19 & $=38.0 \mathrm{ug} / \mathrm{Std}$ & \multirow{10}{*}{$\begin{array}{l}\text { undeutl. Reaktion } \\
15 \mu \mathrm{g} \mathrm{H}_{2} \mathrm{~S} \\
15 ",\end{array}$} \\
\hline & 357 & $=357$ & 36.8 & $=36.8$ & \\
\hline & 545 & $=363.3$ & 56.6 & $=37.7$ & \\
\hline & 755 & $=377.5$ & 76 & $=38.0$ & \\
\hline & 940 & $=376.0$ & 95 & $=38.0$ & \\
\hline & 1172 & $=390.7$ & 115 & $=38.3$ & \\
\hline & 1323 & $=378.0$ & 132 & $=37.7$ & \\
\hline & 1510 & $=377.5$ & 152 & $=38.0$ & \\
\hline & 1660 & $=368.8$ & 167 & $=37.1$ & \\
\hline & Mitt & 1: 377.6 & & $1: 37.7$ & \\
\hline
\end{tabular}

Tabelle 4.

Aus $10 \mathrm{~g} \mathrm{ZnS} \mathrm{der} \mathrm{Korngröße} 2$ (Durchm. $0.90 \mathrm{~mm}$ ) wurden entw. bei $20^{\circ} \mathrm{C}$ :

\begin{tabular}{|c|c|c|c|c|}
\hline Durch $\mathrm{H}_{2} \mathrm{SO}_{4}$ von & $\begin{array}{l}\text { Bei einer } \\
11 / 2 \text { Std. }\end{array}$ & $\begin{array}{c}\text { nwirkungsda } \\
2^{1} / 2 \text { Std. }\end{array}$ & $\begin{array}{l}\text { er von } \\
4^{1 / 2} \mathrm{Std}\end{array}$ & \\
\hline $\begin{array}{l}1.25 \% \\
0.125 \\
0.0125\end{array}$ & $\begin{array}{l}60^{*} \\
-\end{array}$ & $\begin{array}{c}109 \\
\text { undeutl. Reakt. } \\
\text { - }\end{array}$ & $\begin{array}{r}193 \\
20 \\
-\end{array}$ & $\mu \mathrm{g} \quad \mathrm{H}_{2} \mathrm{~S}$ \\
\hline Entwick. $\mathrm{H}_{2} \mathrm{~S}$-Menge & $40^{*}$ & 43.6 & 42.9 & Mittel: $42.2 \mu \mathrm{g} / \mathrm{Std}$. \\
\hline
\end{tabular}

Tabelle 5.

Aus $10 \mathrm{~g} \mathrm{ZnS} \mathrm{der} \mathrm{Korngröße} 3$ (Durchm. $0.75 \mathrm{~mm}$ ) wurden entw. bei $20^{\circ} \mathrm{C}$ :

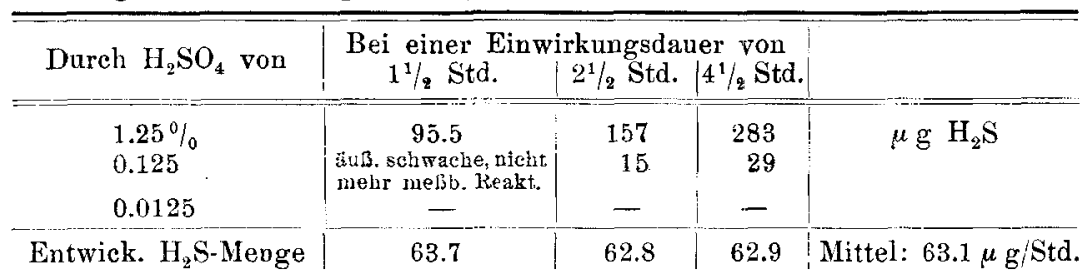

${ }^{1}$ Da $10 \mathrm{~g}$ Erz bei der geringen Angreifbarkeit durch die Säure mitunter etwas zu wenig war, so wurden größere Mengen angewendet; die Proportionalität des Angriffs mit der Oberfläche blieb auch hier erhalten. 
Über die Geschwindigkeit der Auflösung von Zinkblende u. Bleiglanz. 323

Tabelle 6.

Aus $10 \mathrm{~g} \mathrm{ZnS}$ der KorngröBe 1 (Durchmesser $1.13 \mathrm{~mm}$ ) wurden entwickelt bei $\forall=80^{\circ} \mathrm{C}$ :

\begin{tabular}{|c|c|c|c|c|}
\hline Durch $\mathrm{H}_{8} \mathrm{SO}_{4}$ von & $\begin{array}{l}\text { Bei einer } \\
11 / 2 \text { Std. }\end{array}$ & $\begin{array}{l}\text { Einwirkungsd } \\
2^{1} / \mathrm{g} \text { Std. }\end{array}$ & $\begin{array}{l}\text { uer } \\
4 \% \text { Yon }\end{array}$ & \\
\hline $\begin{array}{l}1.25 \% \\
0.125 \\
0.0125\end{array}$ & $\begin{array}{r}545 \\
53 \\
- \\
\end{array}$ & $\begin{array}{r}940 \\
93 \\
- \\
\end{array}$ & $\begin{array}{r}1660 \\
164 \\
15 \\
\end{array}$ & $\begin{array}{c}\mu \mathrm{g} \mathrm{H}_{\mathbf{z}} \mathrm{S} \\
",\end{array}$ \\
\hline $\begin{array}{l}\text { Reartions- } \\
\text { geschwin- } \\
\text { digkeit boi }\end{array}\left\{\begin{array}{l}1.25 \% \text { ig. S. } \\
0.125 \% \text { ig. S. }\end{array}\right.$ & $\begin{array}{r}363.3 \\
35.3\end{array}$ & $\begin{array}{l}376 \\
37.2\end{array}$ & $\begin{array}{r}368.8 \\
36.4\end{array}$ & $\begin{array}{c}\mu \mathrm{g} \mathrm{H}_{2} \mathrm{~S} / \mathrm{Std} \\
\eta\end{array}$ \\
\hline
\end{tabular}

Tabelle 7.

Aus $10 \mathrm{~g} \mathrm{ZnS} \mathrm{der} \mathrm{KorngröBe} 3$ (Durchmesser $0.75 \mathrm{~mm}$ ) wurden entwickelt bei $\vartheta=80^{\circ} \mathrm{C}:$

\begin{tabular}{|c|c|c|c|c|}
\hline Durch $\mathrm{H}_{2} \mathrm{SO}_{4}$ von & \multicolumn{3}{|c|}{ Bei einer Einwirkungsdauer von } & \\
\hline $\begin{array}{l}1.25 \% \\
0.125 \\
0.0125\end{array}$ & $\begin{array}{c}1185 \\
119 \\
-\end{array}$ & $\begin{array}{r}2030 \\
202 \\
18\end{array}$ & $\begin{array}{r}3650 \\
363 \\
33\end{array}$ & $\begin{array}{c}\mu \mathrm{g} \mathrm{H}_{2} \mathrm{~S} \\
" \\
"\end{array}$ \\
\hline $\begin{array}{l}\text { Reaktions- } \\
\text { geschnwin- } \\
\text { digreit bei }\end{array}\left\{\begin{array}{l}0.125 \% \text { ig. S. } \\
\text { ig. S. }\end{array}\right.$ & $\begin{array}{r}790.0 \\
79.3\end{array}$ & $\begin{array}{r}812.0 \\
80.8\end{array}$ & $\begin{array}{r}811.1 \\
80.6\end{array}$ & $\mu \mathrm{g} \mathrm{\textrm {H } _ { 2 } \mathrm { S } / \mathrm { Std }}$ \\
\hline
\end{tabular}

Tabelle 8.

Korngröbenverhältnis (3): (2).

(Die nach Tabelle 4 und 5 entwickelten $\mathrm{H}_{2} \mathrm{~S}$-Nengen verhalten sich umgekehrt wie die Quadrate der Korndurchmesser.)

$$
\begin{aligned}
\text { Theorie: } & \frac{0.75^{2}}{0.90^{2}}=0.695 . \\
\frac{60}{95.5} & =0.629 \\
\frac{109}{157} & =0.695 \\
\frac{193}{283} & =0.682
\end{aligned}
$$

Tabelle 9.

KorngröBenverhältnis (3): (1).

(Die nach Tabelle 6 und 7 entwickelten $\mathrm{H}_{\mathbf{q}} \mathrm{S}$-Mengen verhalten sich umgekehrt wie die Quadrate der Korndurchmesser.)

$$
\begin{array}{cc}
\text { Theorie: }: & \frac{0.75^{2}}{1.13^{2}}=0.440 . \\
\frac{545}{1185}=0.460 & \frac{53}{119}=0.445 \\
\frac{940}{2030}=0.458 & \frac{93}{202}=0.460 \\
\frac{1660}{3650}=0.455 & \frac{164}{363}=0.452
\end{array}
$$


Es ist also die entwickelte Schwefelwasserstoffmenge $y$ :

1. direkt proportional der Zeitdauer $Z$ der Reaktion,

$2 . \quad, \quad, \quad$, Schwefelsäurekonzentration $\left[\mathrm{H}_{2} \mathrm{SO}_{4}\right]$,

3. " " " OberflächengröBe $O$, und endlich

4. " " " absoluten angewandten Menge $M$ des Sulfids, insofern als ja bei einem gleichkörnigen Präparat die Oberfläche der Gewichtsmenge proportional ist, d. h. es ist:

$$
y=f^{\prime} \cdot Z \cdot\left[\mathrm{H}_{2} \mathrm{SO}_{4}\right] \cdot O \cdot M,
$$

worin $f$ einen Proportionalitätsfaktor bedeutet, der außer den Maßeinheiten noch den bisher unberücksichtigt gebliebenen Temperatureinfluß, sowie möglicherweise eine von den besonderen Eigenschaften des Sulfids abhängige Konstante entbält.

Die Versuche, die den TemperatureinfluB auf die Reaktionsgeschwindigkeit ermitteln sollten, führten zu den in den Tabellen 10,

Tabelle 10.

Aus $10 \mathrm{~g} \mathrm{ZnS} \mathrm{der} \mathrm{KorngröBe} 1$ (Durchmesser $=1.13 \mathrm{~mm}$ ) wurden entwickelt von $1.25 \%$ iger $\mathrm{H}_{2} \mathrm{SO}_{4}$ in $1 \%$ Stunden:

\begin{tabular}{c|c|c|c}
\hline Bei $\vartheta={ }^{\circ} \mathrm{C}$ & $y \mu \mathrm{g} \mathrm{H}_{2} \mathrm{~S}$ & $\ln y$ & Temperaturkoeffizient $a$ \\
\hline 0 & $16.7^{*}$ & 2.833 & 0.045 \\
20 & 42 & 3.738 & 0.029 \\
30 & 56 & 4.025 & 0.055 \\
40 & 97 & 4.575 & 0.040 \\
50 & 145 & 4.977 & 0.055 \\
60 & 251 & 5.525 & 0.0415 \\
70 & 380 & 5.940 & 0.036 \\
80 & 545 & 6.301 & \\
\hline
\end{tabular}

Mittel: 0.043

* Mittel aus 3 Bestimmungen: 16, 17 und 17.

Tabelle 11.

Aus $10 \mathrm{~g} \mathrm{ZnS} \mathrm{der} \mathrm{KorngröBe} 3$ (Durchmesser $=0.75 \mathrm{~mm}$ ) wurden entwickelt von $1.25 \%$ iger $\mathrm{H}_{2} \mathrm{SO}_{4}$ in $1 \frac{1}{2}$ Stunden:

\begin{tabular}{c|c|c|c}
\hline Bei $\vartheta={ }^{0} \mathrm{C}$ & $y \mu \mathrm{g} \mathrm{H}_{\mathbf{2}} \mathrm{S}$ & $\ln y$ & Temperaturkoeffizient $a$ \\
\hline 0 & 37.5 & 3.624 & \\
20 & $\mathbf{9 5 . 5}$ & $\mathbf{4 . 5 5 9}$ & 0.047 \\
30 & 138 & 4.927 & 0.037 \\
40 & 210 & 5.347 & 0.042 \\
50 & $\mathbf{3 2 6}$ & $\mathbf{5 . 7 8 7}$ & 0.044 \\
60 & 548 & 6.306 & 0.052 \\
70 & 874 & 6.773 & 0.047 \\
80 & $\mathbf{1 2 7 8}$ & 7.153 & 0038 \\
\end{tabular}


$\ddot{U}$ ber die Geschwindigkeit der Auflösung von Zinkblende u. Bleiglañ. 325

11 u. 12 zusammengestellten und durch die Fig. 1 u. 2 graphisch wiedergegebenen Ergebnissen.

Tabelle 12.

Aus $20 \mathrm{~g} \mathrm{ZnS}$ der Korngröße 2 (Durchmesser $=0.90 \mathrm{~mm}$ ) wurden entwickelt von $0.125 \%$ iger $\mathrm{H}_{2} \mathrm{SO}_{4}$ in $2 \frac{1}{2}$ Stunden:

\begin{tabular}{c|c|c|c}
\hline Bei $\vartheta={ }^{0} \mathrm{C}$ & $y \mu \mathrm{g} \mathrm{H}_{2} \mathrm{~S}$ & $\ln y$ & Temperaturkoeffizient $a$ \\
\hline 0 & 9 & 2.197 & \\
20 & 22 & 3.091 & 0.045 \\
30 & 32 & 3.466 & 0.0375 \\
40 & 48 & 3.871 & 0.041 \\
50 & 76 & 4.331 & 0.046 \\
60 & 127 & 4.844 & 0.051 \\
70 & 200 & 5.298 & 0.045 \\
80 & 294 & 5.684 & 0.0385 \\
& & & Mittel: 0.043
\end{tabular}

Aus Tabelle 10 und 11 folgt:

Tabelle 13.

KorngröBenverbältnis (3): (1).

Theorie: 0.440 .

$$
\begin{aligned}
& \frac{17}{37.5}=0.453 \\
& \frac{42}{95.5}=0.440 \\
& \frac{56}{138}=0.406 \\
& \frac{97}{210}=0.462 \\
& \frac{145}{326}=0.445 \\
& \frac{251}{548}=0.458 \\
& \frac{380}{874}=0.435 \\
& \frac{545}{1278}=0.426 \\
& \text { Mittel }: 0.4406
\end{aligned}
$$

Aus Tabelle 11 und 12 folgt unter Berücksichtigung der Proportionalität zwischen der entwickelten $\mathrm{H}_{2} \mathrm{~S}$-Menge und der Zeitdaner der Reaktion, der Schwefelsäurekonzentration und derabsoluten Menge der angewandten Zinkblende:

\section{Tabelle 14.}

$$
\text { KorngröBenverhältnis (3): (2). }
$$

Theorie: 0.695 .

$$
\begin{aligned}
& \frac{1}{2} \cdot \frac{3}{5} \cdot 10 \cdot \frac{22}{95.5}=0.692 \\
& \cdot \cdot \frac{32}{138}=0.681 \\
& \cdot \quad \cdot \frac{48}{210}=0.686 \\
& . \quad \cdot \frac{76}{326}=0.700 \\
& \cdot \quad \cdot \frac{127}{548}=0.696 \\
& \cdot \quad \cdot \frac{200}{874}=0.686 \\
& . \quad \frac{294}{1278}=0.690 \\
& \text { Mittel }: 0.690
\end{aligned}
$$

Trägt man die Logarithmen der unter sonst gleichen Bedingungen bei verschiedener Temperatur entwickelten Schwefelwasserstoffmengen als Funktion der Temperatur auf Millimeterpapier 
auf (Fig. 3), so erhält man parallele Gerade ${ }^{1}$. Hieraus folgt, da $B$ der EinfluB der Temperatur sich unabhängig von der Korn. größe nach einer Exponentialfunktion ändert, deren allgemeine Gleichung lautet:

$$
y=e^{a \vartheta+b}=k \cdot e^{a \vartheta}
$$

Hierin bedeutet: $y$ die entwickelte $\mathrm{H}_{2} \mathrm{~S}$. Menge, $e$ die Basis des natürlichen Logarithmensystems, $\vartheta$ die Temperatur in Celsiusgraden, $a$ und $k$ noch näher zu bestimmende Konstanten. ${ }^{2}$

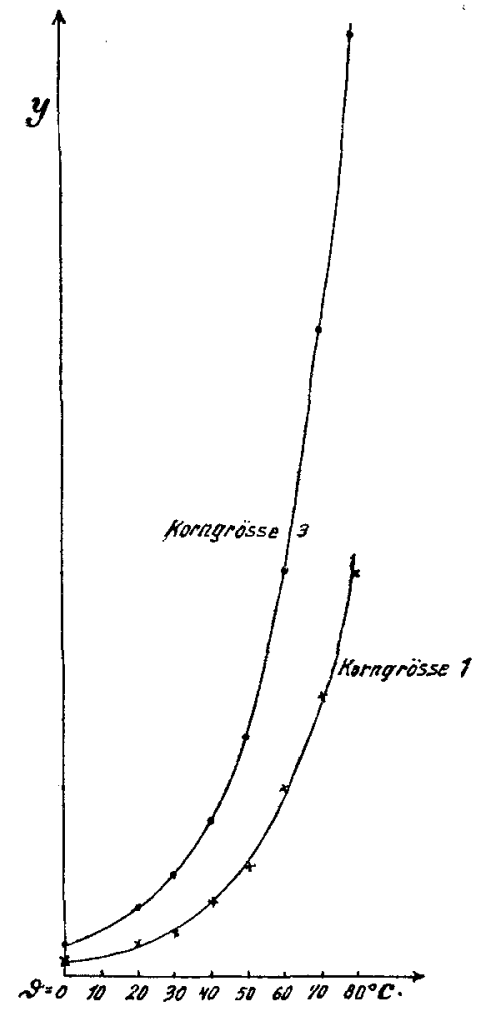

Fig. 1. Die aus $10 \mathrm{~g}$ Clausthaler Blende (KorngröBe 1 u. 3) bei einer Einwirkungsdauer von $1 \frac{1}{2}$ Stunden durch $1.25 \%$ ige $\mathrm{H}_{2} \mathrm{SO}_{4}$ entwickelte Menge $\mathrm{H}_{\mathfrak{z}} \mathrm{S}(y)$ als Funktion der Temperatur $\vartheta$.

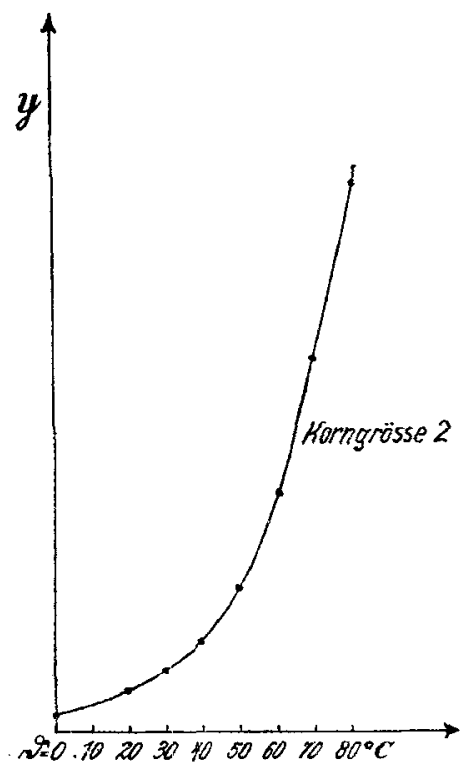

Fig. 2. Die aus $20 \mathrm{~g}$ Clausthaler Blende (Korngröße 2) bei einer Einwirkungsdauer von $2 \% / 2$ Std. durch $0.125 \%$ ige $\mathrm{H}_{2} \mathrm{SO}_{4}$ entwickelte Menge $\mathrm{H}_{2} \mathrm{~S}(y)$ als Funktion der Temperatur $\mathscr{}$.

${ }^{1}$ Die Abweichungen zeigen nicht den geringsten Gang, sondern verteilen sich unregelmäBig auf beide Seiten der Geraden; außerdem liegen sie innerhalb der zulässigen Fehlergrenzen.

${ }^{2}$ In ähnlicher Weise hat bereits früher SPRINa, Zeitschr. phys. Chem. 1 (1887), 209, den Einfluß der Temperatur auf die Reaktionsgeschwindigkeit formuliert. 
Über die Geschwindigkeit der Auflösung von Zinkblende u. Bleiglanz. 327

Aus $y=k \cdot e^{a} \vartheta$ folgt für $\vartheta=0^{0}$ :

$$
y_{0}=k \text {, }
$$

d. h. die Konstante $k$ ist gleich der bei $0^{\circ} \mathrm{C}$ entwickelten Schwefelwasserstoffmenge und natürlich für jede Versuchsreihe verschieden.

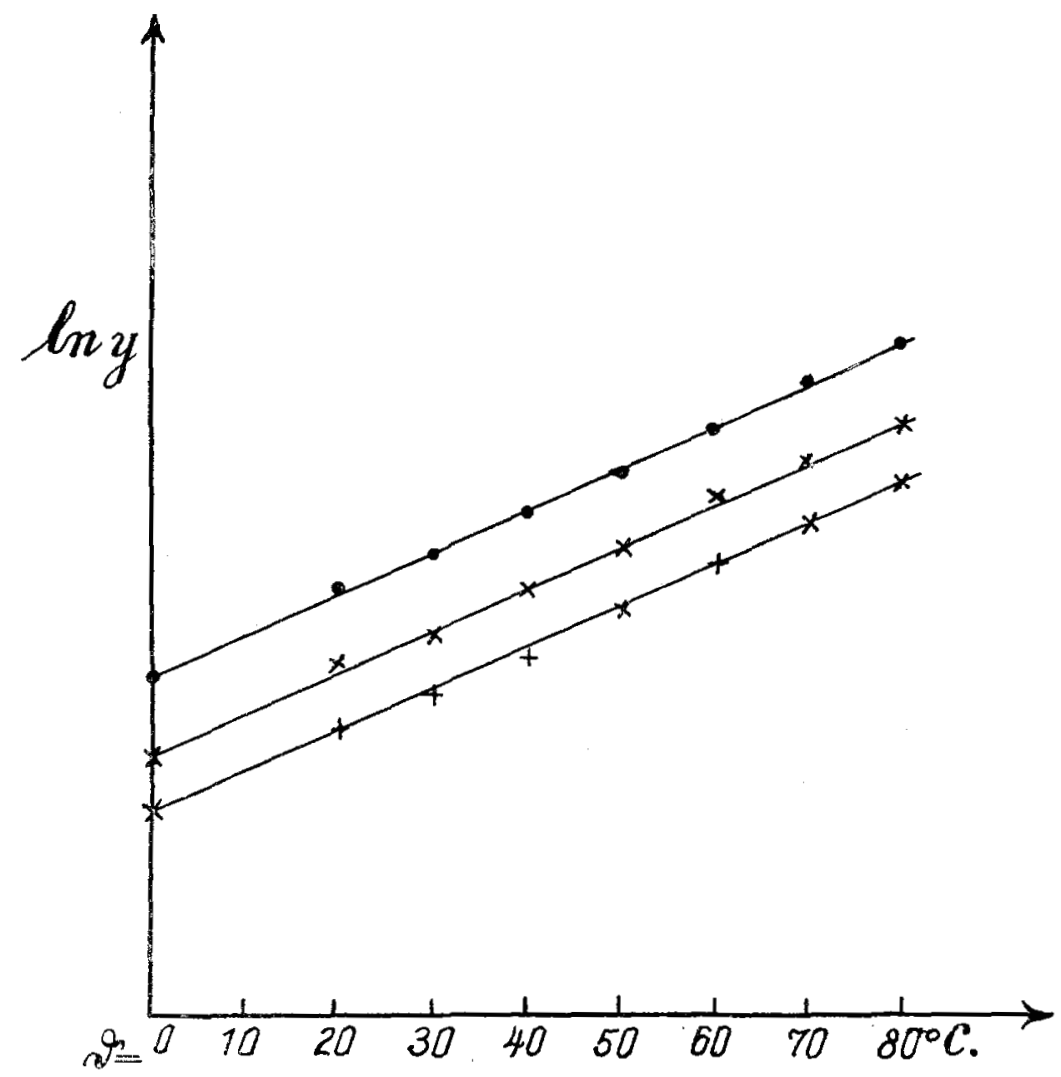

Fig. 3. Die nat. Logaritbmen (ln $y$ ) der nach Tabelle 10,11 u. 12 entwickelten Schwefelwasserstoffmengen als Funktion der Temperatur $\vartheta$. Versuche der Tabelle 10: $x$; Versuche der Tabelle 11: O Versuche der Tabelle 12: +.

Für $a$ ergibt sich: $a=\frac{\ln y_{1}-\ln y_{2}}{\vartheta_{1}-\vartheta_{2}}$, wo $y_{1}$ und $y_{2}$ die bei den Temperaturen $\vartheta_{1}$ und $\vartheta_{2}$ erzeugten Schwefelwasserstoffmengen bedeuten. Die nach dieser Formel berechneten Werte von $a$ sind in den Tabellen mit angeführt; sie stimmen nicht nur innerhalb einer Versuchsreihe hinreichend überein ${ }^{1}$, sondern haben, wie die Paralle-

${ }^{1} a$ läBt sich auch nach der Formel $a=\frac{\ln y-\ln k}{y}$ berechnen, wobei die Konstanz der Werte bedeutend besser ist, doch gibt die im Text angeführte 
lität der drei Geraden in Fig. 3 erkennen läßt und wie auch zu erwarten war, für die drei Versuchsserien innerhalb der Fehlergrenzen den gleichen Wert; als allgemeines Mittel ergibt sich a zu 0.0434.

Die Konstante $k=y_{0}$ der Gleichung ist, wie wir oben gesehen haben:

1. der Zeitdauer $Z$ der Reaktion,

2. der Schwefelsäurekonzentration $\left[\mathrm{H}_{2} \mathrm{SO}_{4}\right]$,

3. der Oberfläche $O$ und

4. der angewandten Gewichtsmenge $M$ des Sulfids direkt proportional, d. h. es ist, wenn die bereits erwähnte Einheitsund Materialkonstante mit $c$ bezeichnet wird, die entwickelte Schwefelwasserstoffmenge

$$
y=e \cdot Z \cdot\left[\mathrm{H}_{2} \mathrm{SO}_{4}\right] \cdot O \cdot M \cdot e^{a \vartheta} .
$$

Tabelle 15.

Aus $20 \mathrm{~g}$ Bleiglanz der Korngröße 1 (Durchmesser $=1.13 \mathrm{~mm}$ ) wurden von $0.125 \%$ iger $\mathrm{H}_{2} \mathrm{SO}_{4}$ bei $80^{\circ} \mathrm{C}$ entwickelt:

\begin{tabular}{c|c|c}
\hline Nach Stdn. & $\mu \mathrm{g} \mathrm{H}_{2} \mathrm{~S}$ & Reaktionsgeschwindigkeit $\mu \mathrm{g} \mathrm{H}_{2} \mathrm{~S} / \mathrm{Std}$ \\
\hline $1 / 2$ & - & - \\
1 & - & - \\
$1^{1 / 2}$ & 10 & 6.66 \\
2 & 13 & 6.50 \\
$2^{1 / 2}$ & 16.5 & 6.60 \\
3 & 20 & 6.66 \\
$3^{1 / 2}$ & $21^{*}$ & $\left.6.00^{*}\right)$ \\
4 & 27 & 6.75 \\
$4^{1 / 2}$ & 30 & 6.66 \\
\hline
\end{tabular}

* Wert wobl fehlerhaft und deshalb bei der Berechnung des Mittels nicht berücksichtigt.

Tabelle 16.

Aus $20 \mathrm{~g}$ Bleiglanz der Korngröße 3 (Durchmesser $=0.75 \mathrm{~mm}$ ) wurden in $21 / 2$ Stdn. von $1.25 \%$ iger Säure entwickelt:

\begin{tabular}{c|c|c|c}
\hline Bei $\vartheta=0 \mathrm{C}$ & $y \mu \mathrm{g} \mathrm{H} \mathrm{H}_{2}$ & $\ln y$ & Temperaturkoeffizient $a$ \\
\hline \hline 0 & 11 & 2.398 & \\
20 & 27.5 & 3.314 & 0.046 \\
30 & 40 & 3.689 & 0.0375 \\
40 & 60 & 4.094 & 0.0405 \\
50 & $\mathbf{9 5}$ & 4.554 & 0.046 \\
60 & $\mathbf{1 5 9}$ & $\mathbf{5 . 0 6 9}$ & 0.0515 \\
70 & 250 & 5.521 & $\mathbf{0 . 0 4 5}$ \\
80 & $\mathbf{3 7 5}$ & 5.927 & 0.0405 \\
& & & \\
\hline
\end{tabular}

Formel, wie schon E. Brunner, Zeitschr. phys. Chem. 47 (1904), 52 bemerkt, ein weit richtigeres Bild von der wirklichen Übereinstimmung, da sie kleine Abweichungen, die dureh die zweite Formel verwischt werden, viel deutlicher hervortreten läBt. 
Ganz analog sind, wie die vorstehenden Tabellen 15, 16 und 17, zeigen, die an Clausthaler Bleiglanz und an Zinkblenden anderer Herkunft erhalten worden sind.

Auch beim Bleiglanz ist nach Tabelle 15 die entwickelte Schwefelwasserstoffmenge proportional der Zeitdauer der Reaktion, die Reaktionsgeschwindigkeit also konstant. Weiter ist $y$ der Schwefelsäurekonzentration proportional, denn aus $20 \mathrm{~g}$ Bleiglanz der Korngröße 2 wurden in $5 \mathrm{Stdn}$. bei $\vartheta=60^{\circ} \mathrm{C}$ von $0.125 \%$ iger Säure 22, von $1.25 \%$ iger dagegen $219 \mu$ g Schwefelwasserstoff entwickelt. Ein Angriff durch $0.0125 \%$ ige Säure konnte selbst unter den günstigsten Versuchsbedingungen nicht mehr einwandfrei nachgewiesen werden. Ferner ist die entwickelte Schwefelwasserstoffmenge auch hier proportional der Oberfläche des Sulfids. Berechnet man nämlich (mit Berücksichtigung der angeführten GesetzmäBigkeiten) aus der nach Tabelle 15 bei $2^{1 / 2}$ stündiger Einwirkung und $80^{\circ} \mathrm{C}$ und der nach Tabelle 16 bei derselben Temperatur gefundenen Schwefelwasserstoffmenge das Oberflächenverhältnis von Korn 3: Korn 1, so findet man:

$$
\frac{10 \cdot 16.5}{375}=0.440
$$

genau wie die Theorie es fordert.

Auch der Temperatureinfluß ändert sich beim Bleiglanz, wie Tabelle 16 zeigt, in gleicher Weise wie bei der Zinkblende nach einer Exponentialfunktion, ja es ist sogar der Temperaturkoeffieient der Reaktion bei beiden Sulfiden innerbalb der Feblergrenzen der gleiche, $a=0.044$ beim Bleiglanz, $a=0.043$ bei der Claustbaler Zinkblende.

Tabelle 17.

Entwickelte Schwefelwasserstoffmenge.

\begin{tabular}{|c|c|c|c|c|c|c|c|c|c|c|}
\hline $\begin{array}{l}\text { Angew. } \\
\text { Gewichts- } \\
\text { mengeing }\end{array}$ & $\begin{array}{c}\text { KorngröBe } \\
\text { (Durch- } \\
\text { messer) }\end{array}$ & in Std. & {$\left[\begin{array}{c}\mathrm{H}_{2} \mathrm{SO}_{4} \\
\text { in } \%\end{array}\right.$} & $\stackrel{\vartheta}{\vartheta^{0} \mathrm{C}}$ & $\begin{array}{r}\text { Blen } \\
\text { Pic } \\
\text { Eu }\end{array}$ & $\begin{array}{l}\text { de von } \\
\text { os de } \\
\text { ropa }\end{array}$ & $\begin{array}{l}\text { Ble } \\
\text { Be } \\
\text { Be }\end{array}$ & $\begin{array}{l}\text { ide von } \\
\text { zelius- } \\
\text { asberg }\end{array}$ & \multicolumn{2}{|c|}{ Christophit } \\
\hline 10 & $13 \mathrm{~mm}$ & & 0.125 & 50 & $7.5^{1}$ & $\mu \mathrm{gH}_{2} \mathrm{~S}$ & .26 & $\mu \mathrm{gH}_{2} \mathrm{~S}$ & 33 & $\mu \mathrm{gH}_{2} \mathrm{~S}$ \\
\hline 10 & $1(1.13$ & $21 / 2$ & 1.25 & 20 & 33 & , & 120 & & 153 & , \\
\hline 10 & 26.90 & $11 / 2$ & 1.25 & 20 & 31 & & 111 & & 146 & \\
\hline 10 & $2(0.90$ & $1 \%$ & 0.125 & 40 & $7.5^{1}$ & " & 26 & & 33 & \\
\hline 10 & .75 & $11 / 2$ & 1.25 & 0 & 19 & & 67 & & 86 & \\
\hline 10 & .75 & $11 / 2$ & 0.125 & 30 & - & & 25 & & 32 & $n$ \\
\hline 10 & $3(0.75$, & $41 / 2$ & 0.125 & 20 & 14 & $"$ & 50 & & 63 & \\
\hline
\end{tabular}

1 Bei diesen Versuchen kamen $20 \mathrm{~g}$ Erz zur Verwendung; die erhaltenen Zahlen wurden nach Division durch 2 in die Tabelle aufgenommen. 
Endlich wurden noch mit einigen uns zur Verfügung stehenden Zinkblenden anderer Herkunft die in der vorstehenden Tabelle 17 verzeichneten Versuche ausgeführt.

An diese experimentellen Daten knüpfte sich folgende Überlegung: Wenn die oben für hiesige Blende und Bleiglanz gefundenen GesetzmäBigkeiten auch für diese Erze gelten, so muß die aus den angeführten Versuchsergebnissen berechnete Temperaturkurve einen konstanten $a$-Wert liefern (der indes nicht mit den früheren übereinzustimmen braucht und selbst bei den verschiedenen Erzen noch verschieden sein kann); ergibt umgekehrt die Rechnung nach obiger Annahme einen konstanten $a$-Wert, so müssen auch die Voraussetzungen der Rechnung, $d$. h. die für Clausthaler Blende und Bleiglanz bewiesenen Gesetzmäßigkeiten Gültigkeit haben. Es wurden also sämtliche in der vorstehenden Tabelle 17 enthaltenen Zahlen unter den angegebenen Voraussetzungen auf die Menge $\mathrm{H}_{2} \mathrm{~S}$ umgerechnet, die aus $10 \mathrm{~g}$ Blende von der Korngröße 3 in $1 \frac{1}{2}$ Stdn. bei der Einwirkung von $1.25 \%$ iger Schwefelsäure bei den verschiedenen Temperaturen entwickelt werden, und aus den erhaltenen Daten die Temperaturkoeffizienten $a$ nach der früher angegebenen Formel berechnet. Die Ergebnisse dieser Rechnungen sind zu Tabelle 18 zusammengestellt:

Tabelle 18.

\begin{tabular}{|c|c|c|c|c|c|c|}
\hline $\begin{array}{l}0 \\
0 \\
I I \\
0\end{array}$ & $\begin{array}{c}y \text { für Blende } \\
\text { vom Picos } \\
\text { de Europa }\end{array}$ & $\begin{array}{c}\text { Tem- } \\
\text { peratur- } \\
\text { koeffizient } \\
a\end{array}$ & $\begin{array}{l}y \text { für Bens- } \\
\text { berger Erz }\end{array}$ & $\begin{array}{c}\text { Tem- } \\
\text { peratur- } \\
\text { koeffizient } \\
a\end{array}$ & $\begin{array}{c}y \text { für } \\
\text { Christophit }\end{array}$ & $\begin{array}{c}\text { Tem- } \\
\text { peratur- } \\
\text { koeffizient } \\
a\end{array}$ \\
\hline \multirow{6}{*}{$\begin{array}{r}0 \\
20 \\
\\
30 \\
40 \\
50\end{array}$} & \multirow{6}{*}{ 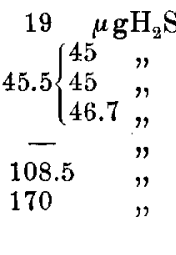 } & \multirow{5}{*}{$\begin{array}{l}0.044 \\
0.0435\end{array}$} & \multirow{6}{*}{ 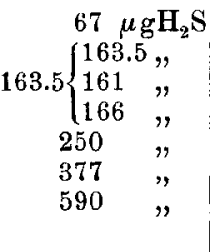 } & & \multirow{6}{*}{ 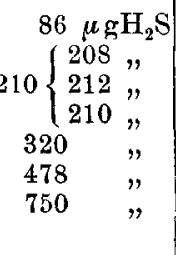 } & \\
\hline & & & & 0.045 & & \\
\hline & & & & 00425 & & 0.042 \\
\hline & & & & 0.04 & & \\
\hline & & & & 0.045 & & \\
\hline & & 0.044 & & 0.043 & & 0.043 \\
\hline
\end{tabular}

Die Übereinstimmung dieser $a$-Werte unter sich und mit den früher gefundenen Werten ist so gut, daß kein Zweifel an der Gültigkeit der besprochenen GesetzmäBigkeiten auch für diese Erze mehr statt hat.

Um dies Ergebnis auch sinnfällig zu machen, wurde nun noch, soweit dies nötig war, je eine Temperaturkurve sämtlicher fünf untersuchter Erze auf gleiche Bedingungen (10 g Erz, KorngröBe 3, 
$1.25 \%$ ige Schwefelsäure, $1 \frac{1}{2}$ Std.) umgerechnet und in ein und dasselbe Koordinatensystem eingetragen (vgl. Fig. 4).

Ein Blick auf die verschiedenen so erhaltenen Kurvenzüge lehrt, daß diese Kurven ,affin" sind, d. h., daB die eine, die abgeleitete Kurve', als aus der anderen, der Einheitskurve, durch Ver- $n$-fachung der Ordinaten hervorgegangen gedacht werden kann. Fassen wir insbesondere die Temperaturkurve der Clausthaler Blende willkürlich als ,Einheitskurve" auf, so erhält man die übrigen, wenn man die Ordinaten der Einheitskurve der Reihe nach beim Christophit mit $n=2.278$, beim Bensberger Erz mit $n=1.780$, bei der Blende von Picos de Europa mit $n=0.5056$ und beim Clausthaler Bleiglanz mit $n=0.0875$ multipliziert. Diese Zahlen $n$, die "Konstanten der abgeleiteten Kurven", geben ohne weiteres an, wieviel mal rascher als die Clausthaler Zinkblende die verschiedenen Erze unter beliebigen, aber gleichen Versuchsbedingungen (gleiche KorngröBe, gleiche Schwefelsäurekonzentration, gleiche Temperatur usw.) angegriffen werden.

\section{Theoretisches.}

Zum SchluB ist die Frage zu erörtern, ob und inwieweit die hier an den sulfidischen Erzen

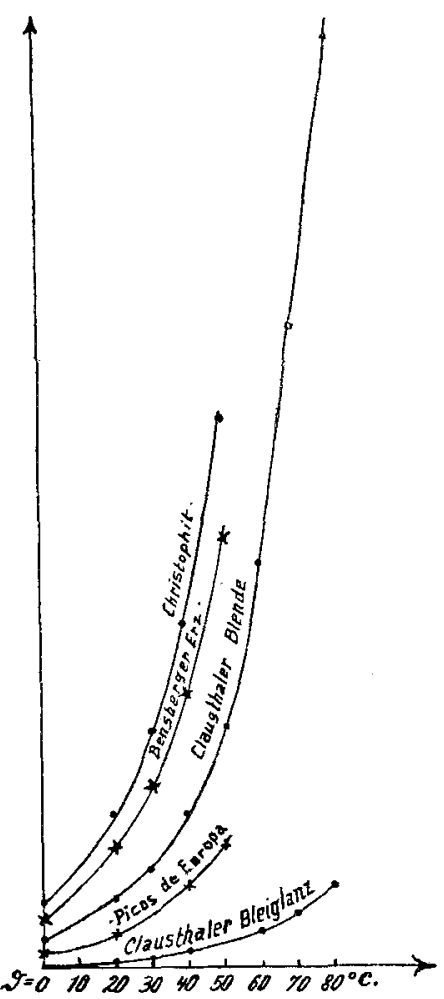

Fig. 4. Die aus $10 \mathrm{~g}$ Erz verschiedener Herkunft (Korngröße 3) in $1 \frac{1}{2}$ Stdn. durch $1.25 \%$ ige $\mathrm{H}_{2} \mathrm{SO}_{4}$ entwickelte Menge $\mathrm{H}_{2} \mathrm{~S}(y)$ als Funktion der Temperatur $\vartheta$. gefundenen GesetzmäBigkeiten mit den Ergebnissen übereinstimmen, die durch die Untersuchungen von Nores und Whitney, von Nernst und Brunner u. A. für die heterogenen Reaktionen gewonnen worden sind. ${ }^{2}$

${ }^{1}$ Die Bezeichnungen wie bei Werner MeckLendora: Über affine Adsorptionskurven, Zeitschr. phys. Chem. 83 (1913), 609.

"Vgl. hierzu: Nernst, ,Theoretische Chemie“, VII. Aufl., Stuttgart 1913, S. $610 \mathrm{ff}$, wo auch die erforderlichen Literaturnachweise zu finden sind. 
Nach Noyes und WhitNey wird die Geschwindigkeit heterogener Reaktionen, die sich ja nur in der Grenze zwischen dem festen Körper und der Flüssigkeit abspielen können, durch die Geschwindigkeit bestimmt, mit welcher der in der Flüssigkeit enthaltene wirksame Stoff an den festen Körper herandiffundiert. Voraussetzung für diese „Diffusionstheorie der heterogenen Reaktionen“ ist die Annahme, dab sich in der Grenzschicht selbst die eigentliche chemische Reaktion mit einer im Vergleich zu der ja verhältnismäBig kleinen Diffusionsgeschwindigkeit großen Geschwindigkeit abspielt. Trifft diese Voraussetzung $\mathrm{zu}$, so ist die gemessene „Reaktionsgesch windigkeit" in Wirklichkeit eine Diffusionsgeschwindigkeit. DaB dies in der Tat der Fall ist, ist an zahlreichen Beispielen erwiesen worden. So fand u. a. BRunner ${ }^{1}$ bei seinen auf Veranlassung von Nernst angestellten Versuchen, daB die Geschwindigkeit der Auflösung von Magnesiumoxyd durch Säuren in erster Linie nicht von der Stärke der Säuren, sondern von ihrer Diffusionsgeschwindigkeit in der Lösung abhängt, und vaN NaMe und seine Mitarbeiter ${ }^{2}$ stellten fest, daß die Geschwindigkeit der Auflösung verschiedener Metalle, $\mathrm{Hg}, \mathrm{Cu}, \mathrm{Ag}, \mathrm{Zn}, \mathrm{Cd}, \mathrm{Fe}, \mathrm{Ni}, \mathrm{Co}$, in wässeriger Jodjodkaliumlösung unter gleichen Bedingungen die gleiche, also unabhängig von der chemischen Natur der Metalle ist und ferner ${ }^{3}$, daß eine Veränderung der inneren Reibung wässeriger Jodjodkaliumlösungen durch Zusatz von Alkohol oder Rohrzucker, durch die ja die Diffusionsgeschwindigkeit geändert wird, eine entsprechende Änderung in der Auflösungsgeschwindigkeit der Metalle zur Folge hat, ein Ergebnis, wie es auch schon von $\mathrm{J}_{\mathrm{ABLCZYNSKI}}$ und $\mathrm{J}_{\mathrm{ABL} \text {.owskI }}{ }^{4}$ bei Versuchen über die Auflösung von Magnesia und Marmor in alkoholhaltiger Salzsäure erhalten worden war.

Bei der Zersetzung sulfidischer Erze durch Schwefelsäure käme nach der Diffusionstheorie als die "Reaktionsgeschwindigkeit" bestimmender Faktor allein die Diffusionsgeschwindigkeit der Säure in Frage. Man sollte also erwarten, daB sich die verschiedenen Erze unter gleichen Bedingungen mit gleicher Geschwindigkeit lösen, während die relativen Lösungsgeschwindigkeiten in Wirklich-

${ }^{1}$ E. Brunner, Zeitschr. phys. Chem. 47 (1904), 52.

2 R. G. van Name und Graham Edgar, Zeitsehr. phys. Chem. 73 (1910), 97.

- van Name und Bosworth, Am. Journ. Sci. [4] 32, 207.

${ }^{8}$ R. G. van Name und D. U. Hill, Z. anorg. Chem. $\$ 6$ (1914), 279.

* R. JABLCZYNSKI und St. JABLOWsKI, Zeitschr. phys. Chem. 75 (1910), 503. 
keit zwischen 0.0875 beim Clausthaler Bleiglanz und 2.278 beim Christophit schwanken.

Die Deutung dieses nach der Theorie nicht erwarteten Ergebnisses könnte man zunächst in der Umkebrbarkeit der beiden Reaktionen

$$
\begin{array}{r}
\mathrm{ZnS}+\mathrm{H}_{2} \mathrm{SO}_{4} \rightleftarrows \mathrm{ZnSO}_{4}+\mathrm{H}_{2} \mathrm{~S} \\
\text { und } \mathrm{PbS}+\mathrm{H}_{2} \mathrm{SO}_{4} \rightleftarrows \mathrm{PbSO}_{4}+\mathrm{H}_{2} \mathrm{~S}
\end{array}
$$

suchen. Indessen beweist die strenge Proportionalität zwischen der Schwefelwasserstoffentwickelung und der Reaktionsdauer, daB die zur Rückbildung der Sulfide führenden Gegenreaktionen keinen Einflub haben, und ein etwa in der Grenzschicht selbst auftretendes stationäres Gleichgewicht könnte die Reaktionsgeschwindigkeit als solche bei der groBen Geschwindigkeit, mit der die nach der einfachen Reaktionsgleichung hier allein in Betracht zu ziehenden Ionenreaktionen zu verlaufen pflegen, kaum beeinflussen; auch wäre in diesem letzten Falle die Proportionalität zwischen dem Angriff der Erze und der Schwefelsäurekonzentration nicht verständlich.

Unter diesen Umständen muB also der SchluB gezogen werden, daB nach den bisher vorliegenden Versuchsdaten die Geschwindigkeit der Einwirkung von verdünnter Schwefelsäure auf Zinkblende und Bleiglanz nicht yon der Diffusionstheorie der Geschwindigkeit heterogener Reaktionen umfaßt wird. Dieser Schlub stimmt mit der Beobachtung überein, daB die Geschwindigkeit der Reaktion nicht, wie es bei den durch Diffusionsvorgänge beherrschten heterogenen Reaktionen der Fall ist, durch Verstärkung des Schüttelns oder Rührens beeinfluBt wird: Durch starke Durchmischung des in Reaktion befindlichen Systems wird wohl - infolge einer Verkleinerung des Diffusionsweges - die Diffusion begünstigt, aber das ist für die gemessene Reaktionsgeschwindigkeit ohne Bedeutung, wenn sie durch die Diffusionsgeschwindigkeit überhaupt nicht bestimmt wird.

Ähnliche Anomalien sind auch schon in anderen Fällen, so z. B. bei der Auflösung von Arsenik in Wasser, beim Schmelzen des Quarzes und bei der Kristallisation aus wässeriger Lösung, beobachtet worden. Da nun bei den Reaktionen in heterogenen Systemen die Diffusion unter allen Umständen eine Rolle spielen muB, so wird man die Deutung derartiger Anomalien wohl meist darin zu suchen haben, dab die von NoYES und WHITNEY gemachte Annahme, nach der die Geschwindigkeit der eigentlichen chemischen Reaktion sehr grob gegenüber der Diffusionsgeschwindigkeit ist, bei ihnen nicht zutrifft. So ist z. B. bei der Auflösung von Arsenik 
in Wasser nach BRUNNER ${ }^{1}$ eine langsam verlaufende Hydratation und beim Schmelzen des Quarzes nach NERNst ein Depolymerisationsvorgang in Betracht zu ziehen. In ähnlicher Weise dürfte es sich auch beim Angriff auf Zinkblende und Bleiglanz durch verdünnte Schwefelsäure um eine, der eigentlichen Umsetzungsreaktion zu Sulfat und Schwefelwasserstoff vorausgehende langsam verlaufende Lösung des Erzes handeln, deren Ursache vielleicht, worauf auch die Gleichheit des Temperaturkoefizienten $a$ für die verschiedenen Erze deutet, in allmählicher Hydrolyse zu suchen ist. In der Tat hat W EIGEL ${ }^{2}$ bei seinen Versuchen, die Löslichkeit von Schwermetallsulfiden in Wasser zu bestimmen, festgestellt, daß die Einstellung des Lösungsgleichgewichtes sowohl beim Bleiglanz als auch bei der Zinkblende erhebliche Zeit erfordert, nämlich je nach der Herkunft des Erzes beim Bleiglanz 20-23, bei der Zinkblende 5-7 Stdn. Nach Weigeus Angaben löst sich die Zinkblende von Santander vier- bis fünfmal schneller als Bleiglanz, während nach unseren Daten das Verbältnis für Clausthaler Bleiglanz und die der Santanderblende sehr nahe stehende Blende ron Picos de Europa 0.0875: $0.5056=1: 5.8$ ist, also ein ganz ähnliches Verhältnis.

Die Frage, warum die Lösungsgeschwindigkeiten der verschiedenen Erze so verschiedene Werte haben, läBt sich zurzeit nicht beantworten. Eine einfache Beziehung zwischen der Lösungsgeschwindigkeit und der in Tabelle 1 dieser Mitteilung verzeichneten Zusammensetzung der Erze ist nicht mit Sicherheit zu erkennen und läßt sich auch aus den Angaben von WEIGEL, der mit natürlichen und synthetischen Erzen von groBer Reinheit, leider aber unbekannter KorngröBe arbeitete, nicht entnehmen. Die von WEIGEL festgestellten Lösungsgeschwindigkeiten gehen nicht einmal den von ihm festgestellten Löslichkeiten parallel, ein Umstand, der wohl nur zum Teil durch verschiedene KorngröBe der verwendeten Erze zu deuten ist. Die weiter oben durch den Buchstaben $n$ bezeichnete relative Lösungsgeschwindigkeit ist also eine auf andere GröBen bislang nicht mit Sicherheit zurückführbare individuelle Konstante der verschiedenen Erze.

1 E. Brunner, Zeitschr. phys. Chem. 51 (1905), 494.

2 Oskar Weiger, „Über das Verhalten von Schwermetallsulfiden in wässeriger Lösung“, Nachr. d. K. Gesellsch. d. Wiss. z. Göttingen, Mathem.-Phygikal. Klasse, 8. Dez. 1906.

Clausthal, Chemisches Laboratorium der Bergakademis, 5. Mai 1914.

Bei der Redaktion eiugegangen am 7. Mai 1914. 